\title{
The binary Goldbach problem with arithmetic weights attached to one of the variables
}

by

\author{
D. I. TOlev (Sofia)
}

1. Introduction and statement of the results. Suppose that $N$ is a sufficiently large integer and denote

$$
J(n)=\sum_{p_{1}+p_{2}=n} \log p_{1} \log p_{2} .
$$

(The letter $p$, with or without subscripts, is reserved for primes.) It is expected that if $n$ is a large even integer then $J(n) \sim c_{0} \lambda(n) n$, where

$$
\lambda(k)=\prod_{\substack{p \mid k \\ p>2}} \frac{p-1}{p-2}, \quad c_{0}=2 \prod_{p>2}\left(1-\frac{1}{(p-1)^{2}}\right) .
$$

This conjecture has not been proved so far, but using the Hardy-Littlewood circle method and Vinogradov's method for estimating exponential sums over primes (see, for example, Vaughan [11, Ch. 2]) one can find that

$$
\sum_{\substack{n \leq N \\ 2 \mid n}}\left|J(n)-c_{0} \lambda(n) n\right| \ll N^{2} \mathcal{L}^{-A},
$$

where $A>0$ is an arbitrarily large constant and $\mathcal{L}=\log N$.

Let $r(k)$ be the number of solutions of the equation $x_{1}^{2}+x_{2}^{2}=k$ in integers $x_{1}, x_{2}$. One of the classical problems in prime number theory is the HardyLittlewood problem concerning the representation of large integers as a sum of two squares and a prime. It was solved by Linnik (see [7]) and related problems have been studied by Linnik, Hooley and other mathematicians. For more information we refer the reader to Hooley's book [5, Ch. 5]. In 
particular, one can show that

$$
\sum_{p \leq N} r(p-1)=\pi N \mathcal{L}^{-1} \prod_{p>2}\left(1+\frac{\chi(p)}{p(p-1)}\right)+O\left(N \mathcal{L}^{-1-\theta_{0}}(\log \mathcal{L})^{5}\right),
$$

where $\chi(k)$ is the non-principal character modulo 4 and

$$
\theta_{0}=\frac{1}{2}-\frac{1}{4} e \log 2=0.0029 \ldots
$$

Let $\tau(k)$ be the number of positive divisors of $k$. Linnik [7] (see also Halberstam and Richert [4, Ch. 3.5]) solved the Titchmarsh divisor problem and proved that

$$
\sum_{p \leq N} \tau(p-1)=c_{1} N+O\left(N \mathcal{L}^{-1} \log \mathcal{L}\right), \quad c_{1}=\prod_{p}\left(1+\frac{1}{p(p-1)}\right) .
$$

We note that sharper versions of (3) and (5) are known at present (see Bredihin [2, Bombieri, Friedlander and Iwaniec [1] and Fouvry [3]).

In this paper we state two theorems which are, in some sense, combinations of (2), (3) and respectively (2), (5). Denote

$$
\mathcal{R}(n)=\sum_{p_{1}+p_{2}=n} r\left(p_{1}-1\right) \log p_{1} \log p_{2} .
$$

After certain formal calculations one may conjecture that for any sufficiently large even $n$ the quantity $\mathcal{R}(n)$ is asymptotically equal to

$$
\begin{aligned}
& \mathcal{M}_{\mathcal{R}}(n) \\
& =\pi c_{0} n \prod_{p \mid n-1}\left(1-\frac{\chi(p)}{p}\right) \prod_{\substack{p \mid n \\
p>2}}\left(1+\frac{p+\chi(p)}{p(p-2)}\right) \prod_{p \nmid n(n-1)}\left(1+\frac{2 \chi(p)}{p(p-2)}\right) .
\end{aligned}
$$

Our first result is the following:

TheOrem 1. Suppose that $\theta_{0}$ is the constant defined by (4). Then

$$
\sum_{\substack{n \leq N \\ 2 \mid n}}\left|\mathcal{R}(n)-\mathcal{M}_{\mathcal{R}}(n)\right| \ll N^{2} \mathcal{L}^{-\theta_{0}}(\log \mathcal{L})^{6} .
$$

It is clear that $n(\log \log (10 n))^{-2} \ll \mathcal{M}_{\mathcal{R}}(n) \ll n(\log \log (10 n))^{2}$. Also, from (8) it follows that for any positive constant $\theta<\theta_{0}$ the number of even $n \leq N$ for which $\left|\mathcal{R}(n)-\mathcal{M}_{\mathcal{R}}(n)\right|>N \mathcal{L}^{-\theta}$ is $O\left(N \mathcal{L}^{-\left(\theta_{0}-\theta\right)}(\log \mathcal{L})^{6}\right)$. So, in other words, $\mathcal{R}(n)$ is close to $\mathcal{M}_{\mathcal{R}}(n)$ for almost all even $n$.

Theorem 1 is related to a recent result of K. Matomäki [8] that the number of integers $n \leq N$ satisfying $n \equiv 0$ or $4(\bmod 6)$ and that cannot be represented as a sum of two primes, one of which of the form $k^{2}+l^{2}+1$, is $O\left(N \mathcal{L}^{-A}\right)$, where $A$ is an arbitrarily large constant. So Matomäki's estimate for the cardinality of this exceptional set is stronger than ours, but her 
method does not provide so sharp information about the number of such representations.

Our second result concerns the quantity

$$
\mathcal{T}(n)=\sum_{p_{1}+p_{2}=n} \tau\left(p_{1}-1\right) \log p_{1} \log p_{2} .
$$

Again, after certain formal calculations, one may conclude that if $2 \mid n$ then $\mathcal{T}(n)$ should be asymptotically equal to

$$
\mathcal{M}_{\mathcal{T}}(n)=c_{0} n \log n \prod_{p \mid n-1}\left(1-\frac{1}{p}\right) \prod_{\substack{p \mid n \\ p>2}}\left(1+\frac{p+1}{p(p-2)}\right) \prod_{p \nmid n(n-1)}\left(1+\frac{2}{p(p-2)}\right) .
$$

We can establish:

THEOREM 2. The following estimate holds:

$$
\sum_{\substack{n \leq N \\ 2 \mid n}}\left|\mathcal{T}(n)-\mathcal{M}_{\mathcal{T}}(n)\right| \ll N^{2}(\log \mathcal{L})^{3} .
$$

We note that

$$
n \log n(\log \log (10 n))^{-2} \ll \mathcal{M}_{\mathcal{T}}(n) \ll n \log n(\log \log (10 n))^{2},
$$

so the quantity $\mathcal{T}(n)$ is close to $\mathcal{M}_{\mathcal{T}}(n)$ for almost all even $n$.

We prove only Theorem 1. The proof of Theorem 2 is similar and simpler.

2. Some lemmas. Suppose that $n \leq N$ and let $k$ and $l$ be integers with $(k, l)=1$ (as usual, $(k, l)$ stands for the greatest common factor of $k$ and $l$ ). Let $\mathcal{I}$ be the set of all subintervals of the interval $[1, N]$ and let $I \in \mathcal{I}$. We denote

$$
J_{k, l}(n ; I)=\sum_{\substack{p_{1}+p_{2}=n \\ p_{1} \equiv l(\bmod k) \\ p_{1} \in I}} \log p_{1} \log p_{2}, \quad J_{k, l}(n)=J_{k, l}(n ;[1, N]) ;
$$

$$
\begin{aligned}
\mathfrak{S}_{k, l}(n) & = \begin{cases}c_{0} \lambda(n k) & \text { if }(k, n-l)=1 \text { and } 2 \mid n, \\
0 & \text { otherwise; }\end{cases} \\
\Phi(n ; I) & =\sum_{\substack{m_{1}+m_{2}=n \\
m_{1} \in I}} 1,
\end{aligned}
$$

and let $\varphi(n)$ be the Euler function.

Our first lemma states that the expected formula for $J_{k, l}(n ; I)$ is true on average with respect to $k \leq \sqrt{N} \mathcal{L}^{-B}$ and $n \leq N$ and uniformly for $l$ and $I$. More precisely, we have 
Lemma 1. For any constant $A>0$ there exists $B=B(A)>0$ such that

$$
\sum_{k \leq \sqrt{N} \mathcal{L}^{-B}} \max _{(l, k)=1} \max _{I \in \mathcal{I}} \sum_{n \leq N}\left|J_{k, l}(n ; I)-\frac{\mathfrak{S}_{k, l}(n)}{\varphi(k)} \Phi(n ; I)\right| \ll N^{2} \mathcal{L}^{-A} .
$$

This lemma is very similar to results of Mikawa [9] and Laporta [6]. These authors study the equation $p_{1}-p_{2}=n$ and without the condition $p_{1} \in I$. However, inspecting the arguments presented in [6], the reader will readily see that the proof of Lemma 1 can be obtained in the same manner.

The next lemma is an immediate consequence of a classical sieve theory result (see [4, Ch. 2, Th. 2.4]).

Lemma 2. Suppose that $h$ is an integer such that $1 \leq|h| \leq N$. Then the number of solutions of the equation $p_{1}-p_{2}=h$ in primes $p_{1}, p_{2} \leq N$ is $O\left(N \mathcal{L}^{-2} \log \mathcal{L}\right)$, where the constant in the Landau symbol is absolute.

The next two lemmas are due to C. Hooley and play an essential role in the proof of (3), as well as in the solutions of other related problems.

Lemma 3. Suppose that $\omega>0$ is a constant and let $F_{\omega}(N)$ be the number of primes $p \leq N$ such that $p-1$ has a divisor lying between $\sqrt{N} \mathcal{L}^{-\omega}$ and $\sqrt{N} \mathcal{L}^{\omega}$. Then

$$
F_{\omega}(N) \ll N \mathcal{L}^{-1-2 \theta_{0}}(\log \mathcal{L})^{3},
$$

where $\theta_{0}$ is defined by (4) and where the constant in the Vinogradov symbol depends only on $\omega$.

Lemma 4. Suppose that $\omega>0$ is a constant. Then

$$
\sum_{p \leq N}\left|\sum_{\substack{d \mid p-1 \\ \sqrt{N} \mathcal{L}^{-\omega}<d<\sqrt{N} \mathcal{L}^{\omega}}} \chi(d)\right|^{2} \ll N \mathcal{L}^{-1}(\log \mathcal{L})^{7},
$$

where the constant in the Vinogradov symbol depends only on $\omega$.

The proofs of very similar results (with $\omega=48$ and with the condition $d \mid N-p$ rather than $d \mid p-1)$ are available in [5, Ch. 5] and the reader will easily see that the method used there also yields the validity of Lemmas 3 and 4 .

\section{Proof of Theorem 1}

3.1. Beginning. Denote by $\mathcal{E}$ the sum on the left-hand side of $(8)$ and put

$$
D=\sqrt{N} \mathcal{L}^{-1-B(1)}
$$


where $B(A)$ is specified in Lemma 1. Using (6) and the well-known identity $r(m)=4 \sum_{d \mid m} \chi(d)$ we find

$$
\mathcal{R}(n)=4 \sum_{p_{1}+p_{2}=n}\left(\sum_{d \mid p_{1}-1} \chi(d)\right) \log p_{1} \log p_{2}=4\left(S_{1}(n)+S_{2}(n)+S_{3}(n)\right),
$$

where

$$
\begin{aligned}
& S_{1}(n)=\sum_{p_{1}+p_{2}=n}\left(\sum_{\substack{d \mid p_{1}-1 \\
d \leq D}} \chi(d)\right) \log p_{1} \log p_{2}, \\
& S_{2}(n)=\sum_{p_{1}+p_{2}=n}\left(\sum_{\substack{d \mid p_{1}-1 \\
D<d<N / D}} \chi(d)\right) \log p_{1} \log p_{2}, \\
& S_{3}(n)=\sum_{p_{1}+p_{2}=n}\left(\sum_{\substack{d \mid p_{1}-1 \\
d \geq N / D}} \chi(d)\right) \log p_{1} \log p_{2} .
\end{aligned}
$$

Therefore from (8) and (13) it follows that

$$
\mathcal{E} \ll \mathcal{E}_{1}+\mathcal{E}_{2}+\mathcal{E}_{3},
$$

where

$$
\mathcal{E}_{1}=\sum_{\substack{n \leq N \\ 2 \mid n}}\left|4 S_{1}(n)-\mathcal{M}_{\mathcal{R}}(n)\right|, \quad \mathcal{E}_{j}=\sum_{\substack{n \leq N \\ 2 \mid n}}\left|S_{j}(n)\right|, \quad j=2,3 .
$$

3.2. Estimation of $\mathcal{E}_{1}$. Using (9), (11), (14) and bearing in mind Lemma 1 we find

$$
S_{1}(n)=\sum_{d \leq D} \chi(d) J_{d, 1}(n)=(n-1) S_{1}^{\prime}(n)+S_{1}^{*}(n),
$$

where

$$
\begin{aligned}
& S_{1}^{\prime}(n)=\sum_{d \leq D} \chi(d) \frac{\mathfrak{S}_{d, 1}(n)}{\varphi(d)}, \\
& S_{1}^{*}(n)=\sum_{d \leq D} \chi(d)\left(J_{d, 1}(n)-(n-1) \frac{\mathfrak{S}_{d, 1}(n)}{\varphi(d)}\right) .
\end{aligned}
$$

Hence

$$
\mathcal{E}_{1} \ll \mathcal{E}_{1}^{\prime}+\mathcal{E}_{1}^{*},
$$

where

$$
\mathcal{E}_{1}^{\prime}=\sum_{\substack{n \leq N \\ 2 \mid n}}\left|4(n-1) S_{1}^{\prime}(n)-\mathcal{M}_{\mathcal{R}}(n)\right|, \quad \mathcal{E}_{1}^{*}=\sum_{\substack{n \leq N \\ 2 \mid n}}\left|S_{1}^{*}(n)\right| .
$$


By (12), 20, 22 and Lemma 1 it follows that

$$
\mathcal{E}_{1}^{*} \ll N^{2} \mathcal{L}^{-1} \text {. }
$$

Consider $\mathcal{E}_{1}^{\prime}$. From (1), 10) and 190 we find

$$
S_{1}^{\prime}(n)=c_{0} \sum_{\substack{d \leq D \\(d, n-1)=1}} \frac{\chi(d)}{\varphi(d)} \lambda(n d)=c_{0} \lambda(n) \sum_{\substack{d \leq D \\(d, n-1)=1}} f_{n}(d),
$$

where

$$
f_{n}(d)=\frac{\chi(d)}{\varphi(d)} \frac{\lambda(d)}{\lambda((n, d))} .
$$

Obviously the function $f_{n}(d)$ is multiplicative with respect to $d$ and

$$
f_{n}(d) \ll d^{-1}(\log \log (10 d))^{2}
$$

uniformly with respect to $n$. To evaluate the sum on the right-hand side of (24) we consider the function

$$
F_{n}(s)=\sum_{\substack{d=1 \\(d, n-1)=1}}^{\infty} f_{n}(d) d^{-s}
$$

It is analytic in the half-plane $\operatorname{Re}(s)>0$ and we may represent it as an Euler product:

$$
F_{n}(s)=\prod_{p \nmid n-1} T_{n}(p, s), \quad T_{n}(p, s)=1+\sum_{l=1}^{\infty} f_{n}\left(p^{l}\right) p^{-l s} .
$$

From (1) and 25) we easily find

$$
f_{n}\left(p^{l}\right)= \begin{cases}\chi(p)^{l} p^{1-l}(p-1)^{-1} & \text { if } p \mid n, \\ \chi(p)^{l} p^{1-l}(p-2)^{-1} & \text { if } p \nmid n ;\end{cases}
$$

and respectively

$$
T_{n}(p, s)=\left(1-\frac{\chi(p)}{p^{s+1}}\right)^{-1} T_{n}^{*}(p, s),
$$

where

$$
T_{n}^{*}(p, s)= \begin{cases}1+\chi(p) p^{-s-1}(p-1)^{-1} & \text { if } p \mid n, \\ 1+2 \chi(p) p^{-s-1}(p-2)^{-1} & \text { if } p \nmid n .\end{cases}
$$

Therefore

$$
F_{n}(s)=L(s+1, \chi) H_{n}(s)
$$

where $L(s, \chi)$ is the Dirichlet $L$-function corresponding to the character $\chi$ 
and

$$
\begin{aligned}
H_{n}(s)= & \prod_{p \mid n-1}\left(1-\frac{\chi(p)}{p^{s+1}}\right) \prod_{p \mid n}\left(1+\frac{\chi(p)}{p^{s+1}(p-1)}\right) \\
& \times \prod_{p \nmid n(n-1)}\left(1+\frac{2 \chi(p)}{p^{s+1}(p-2)}\right) .
\end{aligned}
$$

From 27 and 28 we see that $F_{n}(s)$ has an analytic continuation to the half-plane $\operatorname{Re}(s)>-1$. It is clear that $H_{n}(s) \ll n^{\varepsilon}$ for $|\operatorname{Re}(s)| \geq-1 / 2$ (here and later, $\varepsilon$ is an arbitrarily small positive number). Also, it is well-known that in the same region we have $L(s+1, \chi) \ll 1+|\operatorname{Im}(s)|^{1 / 6}$. Hence

$$
F_{n}(s) \ll N^{\varepsilon} T^{1 / 6} \quad \text { if } \quad \operatorname{Re}(s) \geq-1 / 2, \quad|\operatorname{Im}(s)| \leq T
$$

for any $T>1$. We apply Perron's formula (see, for example [10, Ch. II.2]) to find

$$
\sum_{\substack{d \leq D \\(d, n-1)=1}} f_{n}(d)=\frac{1}{2 \pi i} \int_{\varkappa-i T}^{\varkappa+i T} F_{n}(s) \frac{D^{s}}{s} d s+O\left(\sum_{d=1}^{\infty} \frac{D^{\varkappa}\left|f_{n}(d)\right|}{d^{\varkappa}\left(1+T\left|\log \frac{D}{d}\right|\right)}\right)
$$

with $\varkappa=1 / 10$ and $T=N^{3 / 4}$. Using $(12)$ and $(26)$ one can easily verify that the remainder term in $(30)$ is $O\left(N^{-1 / 20}\right)$. To evaluate the integral in 30 we apply Cauchy's theorem. The residue of the integrand at $s=0$ equals

$$
F_{n}(0)=\frac{\pi}{4} \prod_{p \mid n-1}\left(1-\frac{\chi(p)}{p}\right) \prod_{p \mid n}\left(1+\frac{\chi(p)}{p(p-1)}\right) \prod_{p \nmid n(n-1)}\left(1+\frac{2 \chi(p)}{p(p-2)}\right) .
$$

Hence the main term on the right-hand side of 30 is equal to

$$
F_{n}(0)+\frac{1}{2 \pi i}\left(\int_{\varkappa-i T}^{-1 / 2-i T}+\int_{-1 / 2-i T}^{-1 / 2+i T}+\int_{-1 / 2+i T}^{\varkappa+i T}\right) F_{n}(s) \frac{D^{s}}{s} d s .
$$

Using 29 one can easily find that the contribution of the integrals in 32 is $O\left(N^{-1 / 20}\right)$. Therefore

$$
\sum_{\substack{d \leq D \\(d, n-1)=1}} f_{n}(d)=F_{n}(0)+O\left(N^{-1 / 20}\right) .
$$

From (1), (7), 22), 24), (31) and (33) it follows that

$$
\mathcal{E}_{1}^{\prime} \ll N^{2} \mathcal{L}^{-1} \text {. }
$$

Hence, using (21) and 23) we get

$$
\mathcal{E}_{1} \ll N^{2} \mathcal{L}^{-1}
$$


3.3. Estimation of $\mathcal{E}_{2}$. Clearly, from 18 and Cauchy's inequality it follows that

$$
\mathcal{E}_{2} \ll N^{1 / 2}\left(\sum_{n \leq N}\left|S_{2}(n)\right|^{2}\right)^{1 / 2}=N^{1 / 2}\left(\mathcal{E}_{2}^{\prime}\right)^{1 / 2},
$$

say. Using (15) we find

$$
\begin{aligned}
\mathcal{E}_{2}^{\prime} & =\sum_{n \leq N} \sum_{D<d, t<N / D} \chi(d) \chi(t) \sum_{\substack{p_{1}+p_{2}=n \\
p_{1} \equiv 1(\bmod d)}} \log p_{1} \log p_{2} \sum_{\substack{p_{3}+p_{4}=n \\
p_{3} \equiv 1(\bmod t)}} \log p_{3} \log p_{4} \\
& =\sum_{p_{1}+p_{2}=p_{3}+p_{4} \leq N} \log p_{1} \log p_{2} \log p_{3} \log p_{4} \sum_{\substack{D<d, t<N / D \\
d\left|p_{1}-1, t\right| p_{3}-1}} \chi(d) \chi(t) \\
& \ll \mathcal{L}^{4} \mathcal{E}_{2}^{\prime \prime}+N^{2+\varepsilon},
\end{aligned}
$$

where

$$
\mathcal{E}_{2}^{\prime \prime}=\sum_{\substack{p_{1}+p_{2}=p_{3}+p_{4} \\ p_{1}, p_{2}, p_{3}, p_{4} \leq N \\ p_{1} \neq p_{3}}}\left|\sum_{\substack{D<d<N / D \\ d \mid p_{1}-1}} \chi(d)\right|\left|\sum_{\substack{D<t<N / D \\ t \mid p_{3}-1}} \chi(t)\right| .
$$

Denote by $\mathcal{F}$ the set of primes $p \leq N$ such that $p-1$ has a divisor lying between $D$ and $N / D$. Using the inequality $u v \leq u^{2}+v^{2}$ and taking into account the symmetry with respect to $d$ and $t$ we get

$$
\begin{aligned}
\mathcal{E}_{2}^{\prime \prime} & \ll \sum_{\substack{p_{1}+p_{2}=p_{3}+p_{4} \\
p_{1}, p_{2}, p_{4} \leq N \\
p_{1} \neq p_{3}, p_{3} \in \mathcal{F}}}\left|\sum_{\substack{D<d<N / D \\
d \mid p_{1}-1}} \chi(d)\right|^{2} \\
& =\sum_{p_{1} \leq N}\left|\sum_{\substack{D<d<N / D \\
d \mid p_{1}-1}} \chi(d)\right|^{2} \sum_{\substack{p_{3} \in \mathcal{F} \\
p_{3} \neq p_{1}}} \sum_{\substack{p_{2}, p_{4} \leq N \\
p_{4}-p_{2}=p_{1}-p_{3}}} 1 .
\end{aligned}
$$

Applying Lemmas 2 and 3 we find

$$
\sum_{\substack{p_{3} \in \mathcal{F} \\ p_{3} \neq p_{1}}} \sum_{\substack{p_{2}, p_{4} \leq N \\ p_{4}-p_{2}=p_{1}-p_{3}}} 1 \ll N \mathcal{L}^{-2}(\log \mathcal{L}) \sum_{p \in \mathcal{F}} 1 \ll N^{2} \mathcal{L}^{-3-2 \theta_{0}}(\log \mathcal{L})^{4}
$$

and then using (37), (38) and Lemma 4 we get

$$
\mathcal{E}_{2}^{\prime \prime} \ll N^{2} \mathcal{L}^{-3-2 \theta_{0}}(\log \mathcal{L})^{4} \sum_{p \leq N}\left|\sum_{\substack{D<d<N / D \\ d \mid p-1}} \chi(d)\right|^{2} \ll N^{3} \mathcal{L}^{-4-2 \theta_{0}}(\log \mathcal{L})^{11}
$$

From (35), (36) and (39) we conclude that

$$
\mathcal{E}_{2} \ll N^{2} \mathcal{L}^{-\theta_{0}}(\log \mathcal{L})^{6} .
$$


3.4. Estimation of $\mathcal{E}_{3}$. From 16 it follows that

$$
\begin{aligned}
S_{3}(n) & =\sum_{p_{1}+p_{2}=n} \log p_{1} \log p_{2} \sum_{\substack{m \mid p_{1}-1 \\
\left(p_{1}-1\right) / m \geq N / D}} \chi\left(\frac{p_{1}-1}{m}\right) \\
& =\sum_{p_{1}+p_{2}=n} \log p_{1} \log p_{2} \sum_{j= \pm 1} \chi(j) \sum_{\substack{m \leq\left(p_{1}-1\right) D / N, 2 \mid m \\
p_{1} \equiv 1+j m(\bmod 4 m)}} 1 .
\end{aligned}
$$

We change the order of summation and use (9) to find

$$
S_{3}(n)=\sum_{\substack{m \leq D \\ 2 \mid m}} \sum_{j= \pm 1} \chi(j) J_{4 m, 1+j m}\left(n, I_{m}\right),
$$

where $I_{m}$ denotes the interval $[1+m N / D, N]$. Having in mind Lemma 1 we write

$$
S_{3}(n)=S_{3}^{\prime}(n)+S_{3}^{*}(n),
$$

where

$$
\begin{aligned}
& S_{3}^{\prime}(n)=\sum_{\substack{m \leq D \\
2 \mid m}} \sum_{j= \pm 1} \chi(j) \frac{\mathfrak{S}_{4 m, 1+j m}(n)}{\varphi(4 m)} \Phi\left(n, I_{m}\right) \\
& S_{3}^{*}(n)=\sum_{\substack{m \leq D \\
2 \mid m}} \sum_{j= \pm 1} \chi(j)\left(J_{4 m, 1+j m}\left(n, I_{m}\right)-\frac{\mathfrak{S}_{4 m, 1+j m}(n)}{\varphi(4 m)} \Phi\left(n, I_{m}\right)\right) .
\end{aligned}
$$

Since $2 \mid n$ it follows from $10 \mid$ that

$$
\mathfrak{S}_{4 m, 1+j m}(n)= \begin{cases}c_{0} \lambda(4 m n) & \text { if }(4 m, n-1-j m)=1, \\ 0 & \text { otherwise. }\end{cases}
$$

However, the condition $(4 m, n-1-j m)=1$ is independent of $j$ (from the set $\{1,-1\})$ and therefore $\mathfrak{S}_{4 m, 1+j m}(n)$ is independent of $j$ too. This means that

$$
S_{3}^{\prime}(n)=0 .
$$

Hence, using 12, 18, 41, 42 and Lemma 1 we find

$$
\begin{aligned}
\mathcal{E}_{3} & \ll \sum_{n \leq N}\left|S_{3}^{*}(n)\right| \\
& \ll \sum_{\substack{m \leq D \\
2 \mid m}} \sum_{j= \pm 1} \sum_{n \leq N}\left|J_{4 m, 1+j m}\left(n, I_{m}\right)-\frac{\mathfrak{S}_{4 m, 1+j m}(n)}{\varphi(4 m)} \Phi\left(n, I_{m}\right)\right| \\
& \ll \sum_{k \leq 4 D} \max _{(l, k)=1} \max _{I \in \mathcal{I}} \sum_{n \leq N}\left|J_{k, l}(n, I)-\frac{\mathfrak{S}_{k, l}(n)}{\varphi(k)} \Phi(n, I)\right| \ll N^{2} \mathcal{L}^{-1} .
\end{aligned}
$$


The estimate (8) follows from (17), (34), (40) and (43), so the theorem is proved.

Acknowledgments. The research was supported by Sofia University Grant 028/2009.

\section{References}

[1] E. Bombieri, J. Friedlander and H. Iwaniec, Primes in arithmetic progressions to large moduli II, Math. Ann. 277 (1987), 361-393.

[2] B. Bredihin, The dispersion method and binary additive problems, Russian Math. Surveys 20 (1965), 85-125.

[3] E. Fouvry, Sur le problème des diviseurs de Titchmarsh, J. Reine Angew. Math. 357 (1985), 51-76.

[4] H. Halberstam and H.-E. Richert, Sieve Methods, Academic Press, 1974.

[5] C. Hooley, Applications of Sieve Methods to the Theory of Numbers, Cambridge Univ. Press, 1976.

[6] M. Laporta, A short intervals result for $2 n$-twin primes in arithmetic progressions, Tsukuba J. Math. 23 (1999), 201-214.

[7] Yu. V. Linnik, The Dispersion Method in Binary Additive Problems, Izdat. Leningrad. Univ., Leningrad, 1961 (in Russian).

[8] K. Matomäki, The binary Goldbach problem with one prime of the form $p=$ $k^{2}+l^{2}+1$, J. Number Theory 128 (2008), 1195-1210.

[9] H. Mikawa, On prime twins in arithmetic progressions, Tsukuba J. Math. 16 (1992), $377-387$.

[10] G. Tenenbaum, Introduction to Analytic and Probabilistic Number Theory, Cambridge Univ. Press, 1995.

[11] R. Vaughan, The Hardy-Littlewood Method, Cambridge Univ. Press, 1997.

D. I. Tolev

Faculty of Mathematics and Informatics

Sofia University "St. Kl. Ohridsky"

5 J. Bourchier, 1164 Sofia, Bulgaria

E-mail: dtolev@fmi.uni-sofia.bg

Received on 18.3.2009

and in revised form on 2.8.2009 